\title{
Apuntes para la problematización: ¿desmercantilización o re- mercantilización de la política social actual?: Transferencias estatales y consumo como aspectos a considerar ${ }^{*}$
}

\author{
Andrea Dettano**

\section{Resumen} \\ El presente trabajo busca articular la literatura sobre las esferas \\ de la provisión de Bienestar en pos de hacer una lectura \\ focalizada sobre la política social actual y la esfera de la \\ desmercantilización. En este sentido es que se problematiza y \\ cuestiona el potencial desmercantilizador de la Asignación \\ Universal por Hijo para la Protección social (AUH) -que funciona \\ en Argentina desde 2009-, por medio de un recorrido teórico, \\ reflexionando sobre el concepto de Bienestar, el Estado de \\ Bienestar y sus regímenes. \\ La política bajo análisis es una de las denominadas de "Inclusión \\ Social". Dada su fuerte presencia en América Latina y el Caribe - \\ en 2012 ya se aplican en 18 países alcanzando a cubrir a \\ aproximadamente 129 millones de personas (Stampini y \\ Tornarolli, 2012)-, es pertinente comenzar a indagar sobre la \\ forma en que dichas políticas, de gran alcance a nivel nacional, \\ se convierten en uno más, de los disponibles actualmente, \\ incentivos al consumo. Para esto se recuperan algunos aportes \\ que abonan la articulación analítica entre Políticas sociales y \\ consumo.
}

\section{Palabras clave}

Políticas sociales - Desmercantilización - Bienestar.

\footnotetext{
* Artículo recibido el 28 de Septiembre de 2015. Aceptado el 22 de diciembre de 2015.

** UBA - CONICET - CIES. Correo electrónico: andreadettano@gmail.com
} 


\section{Abstract}

This paper aims to build an articulation between the literature on the welfare provision spheres, the one of decommodification and the current of social policies. In this sense, we dispute, question and promote the analysis of the potential decommodification of AUH (Universal Allocation per Child for Social Protection, in force since 2009) through a theoretical path, reflecting upon the concept of Welfare, Welfare State and their regimes.

The policy under analysis is one of the so-called "Social Inclusion" ones. Bearing in mind their strong presence in Latin America and the Caribbean -in 2012 they were applied in 18 countries almost embracing about 129 million people (Stampini and Tornarolli, 2012) - it is relevant to start investigating how these far-reaching policies at a national level turn into one more of the currently available consumption incentives. To this end, we recover some contributions to delve into the analytical link between social policies and consumption.

\section{Keywords}

Social Policies - decommodification - Welfare-

\section{Introducción}

"Cuando usamos el término 'Política social', no debemos, por consiguiente, reaccionar invistiéndolo automáticamente con un halo de altruismo, preocupación o interés por los otros, por la igualdad, entre otras cosas. Tampoco debemos concluir sin pensar que porque

Gran Bretaña -o cualquier otro país- tiene una política social o ha desarrollado servicios sociales, estos operan de hecho para alcanzar como fines la redistribución, la igualdad y el altruismo. Lo que es 'Bienestar' para algunos grupos, puede ser 'malestar' para otros" (Titmuss, 1974: 127). ${ }^{1}$

El paso de una sociedad tradicional a una moderna (Polanyi, Dobb, Hobsbawm) presenta como problema de análisis la mercantilización de crecientes y variados aspectos de la vida. Pensar en la satisfacción y el bienestar en una sociedad de mercado nos remite indefectiblemente a indagar, en primer lugar, las formas de su provisión, sobre quién, quiénes o qué órbitas recae $y$, en segundo lugar, cómo la definición misma de bienestar se va modificando históricamente.

1 Traducción propia. 
Este escrito busca articular literatura clásica sobre la provisión de Bienestar y sus esferas, poniendo el acento en las formas que cobra la política social actual -como Programas de Transferencias Condicionadas de Ingreso (PTCI)- y cuestionando su posible efecto desmercantilizador. La revisión bibliográfica permite, a modo de antesala para esta reflexión, tomar en cuenta las particularidades de los Regímenes de Bienestar en América Latina, así como los efectos de la aplicación de medidas neoliberales en la estructura del mercado de trabajo y en la política social.

Toda la evidencia recolectada y brevemente expuesta, intenta repensar si es posible hablar de un efecto desmercantilizador de la política social -de la Asignación Universal por Hijo para la Protección Social (AUH), en particular-, partiendo de las dimensiones propuestas por Esping Andersen, y tratando de incorporar nuevos ejes para el análisis. En este sentido, desde aquí consideramos trabajos que hacen referencia a determinadas 'consecuencias' de la política social, como el conjunto de operaciones financieras, endeudamiento e incentivos al consumo producto de las transferencias de dinero a los quintiles más bajos de la población. De este modo, la construcción y ejecución de políticas públicas y sus 'efectos' son el sustrato para pensar y re-pensar el vínculo entre política social, consumo y estructura social.

La estrategia argumentativa del escrito será la siguiente: en primer lugar, se hará una descripción sobre el contexto en el cual surge la $\mathrm{AUH}$; en segundo lugar, se reflexionará sobre el concepto de Bienestar, el Estado de Bienestar y sus regímenes; en tercer lugar, se abordará la relación entre la provisión de Bienestar y su posible efecto desmercantilizador, significado e implicancias; en cuarto lugar, se analizará el potencial desmercantilizador de la $\mathrm{AUH}$ en particular. Por último, se sintetizarán algunas reflexiones finales.

\section{Sobre el contexto}

Reflexionando sobre la recursividad entre estructura social y políticas sociales es que debemos pensar en las modificaciones que la aplicación de políticas neoliberales ha ido delineando en Argentina en las últimas décadas del siglo veinte. Grandes transformaciones en el patrón productivo y de acumulación fueron teniendo lugar desde mediados de los 70, generando un fuerte empobrecimiento de la población que se visualiza en la ampliación de la brecha distributiva. De esta forma, sobre el pasado cuarto de siglo, podemos sostener que: "La combinación entre neoliberalismo, doctrina de la seguridad nacional y autoritarismo inauguró un modelo de apertura económica con perfil agroexportador que, con algunas diferencias, se consolida en la década de los '90 y continúa hasta el día de hoy..." (Scribano, 2008: 2). A la vez, este período, puede considerarse como "el proceso político de producción de la realidad de la pobreza y el desempleo" (Grassi; Alayón, 2005: 112), el cual supone una situación signada por la subordinación del trabajo al dominio del capital, acrecentando los contextos y condiciones de pobreza. Dicha subordinación implica para Grassi y Alayón 
(2005) el desenvolvimiento de una tensión, siempre irresoluble, que puede ser contenida o agudizada por arreglos políticos transitorios, como la política social. A partir de aquí intentaremos ver sus transformaciones y/o continuidades.

Desde las Ciencias Sociales, los noventa llevan la etiqueta del período donde la aplicación de medidas de corte neoliberal llega al límite con las crisis de finales de 2001, dejando un saldo creciente de desigualdades. La década se inicia con la presidencia de Carlos Menem y la adopción, por parte de ese gobierno, de un paquete de medidas acordes al llamado Consenso de Washington. Las medidas económicas aplicadas consistieron en el establecimiento de un tipo de cambio fijo y bajo, la apertura importadora de la economía, privatización de las empresas públicas, desregulación de los mercados -el laboral incluido- y descentralización de los servicios públicos de salud y educación. En cuanto a indicadores de empleo, pobreza y desigualdad distributiva, los rasgos principales de los noventa consisten en un creciente nivel de pobreza, que alcanzó a un $30 \%$ de la población, la disparidad en la distribución del ingreso, que se acentúa por regiones donde el ingreso per cápita en las jurisdicciones más ricas es 4.1 veces superior a las más pobres. A finales de la década el nivel de desempleo llega al $18 \%$ y la informalidad laboral alcanza al $40 \%$ de los empleados en relación de dependencia. La precarización, la caída de los salarios, la subocupación son los rasgos que asume el mercado laboral y se constituye como la cuestión sobresaliente del período (Acuña, Kessler, Repetto, 2002). Sumado a las modificaciones que sufre la legislación en materia de empleo, con la precarización de las condiciones de vida de grandes contingentes de población que eso implica, Argentina comparte con otros países de América Latina la "paradoja del crecimiento sin empleo." Mientras que el Producto Bruto Interno (PBI) aumentaba, el desempleo se mantenía elevado. Desempleo, sobreempleo y empleo por fuera de la ley fueron las modalidades que Grassi y Alayón (2005) enumeraron como los rasgos que asume el mercado laboral de los noventa.

Estos rasgos traen aparejado un retroceso en cuanto a las protecciones sociales, ya que esa población no accede a los beneficios del asalariado formal. Para 1997 solo el $30 \%$ de la población económicamente activa se encontraba protegido por riesgos relacionados con su vida presente como accidentes de trabajo, a la vez que esta población descubierta probablemente encontraría dificultades a la hora de jubilarse (Grassi y Alayón, 2005). Lo expuesto manifiesta, una vez más, que la protección social se erige sobre la condición de asalariado formal, por lo que, al modificarse el mercado de trabajo y sus condiciones de funcionamiento, dicha protección no llega a alcanzar los niveles de cobertura necesarios.

El esquema de seguridad social, en sus cuatro componentes -previsional, obras sociales, asignaciones familiares y riesgos de trabajo- vio reducirse, tal como expresan Hintze y Danani (2013), su cobertura en términos de la cuantía de la población cubierta, así como estableciendo 'máximos' a la protección. A la vez, se introdujeron elementos 
de protección privados -sistema previsional, de salud, riesgos del trabajo- dejándola librada a las fuerzas del mercado, acrecentando su orientación privatista y dando lugar a "un retroceso distributivo y de la solidaridad" (Hintze y Danani, 2013: 46).

Concomitantemente, las políticas de asistencia a la pobreza y el desempleo tuvieron como principal destino la focalización. De este modo:

...la máxima mercantilización de la fuerza de trabajo y la desestructuración de las instituciones de regulación de su uso y de protección de los trabajadores fue correlativa y determinante de la asistencialización y filantropización de la política social, cuyo sujeto no es otro que el trabajador desprotegido, efectiva o potencialmente pobre (Grassi y Alayón; 2005: 125).

Como ya se ha mencionado, es en esta población empobrecida que se focalizaron los planes de asistencia y de promoción del empleo. Las políticas de transferencia de dinero o bienes que tuvieron lugar exigían una contraprestación laboral a sus destinatarios, constituyéndose como "planes de empleo subsidiado" (Borghini et al., 2012).

La crisis de 2001 significó el estallido de las condiciones que se venían gestando en las décadas previas y que se han descripto en párrafos anteriores. En este marco, en 2002 se declara, por medio de la Ley N.²5561, la emergencia ocupacional, social, económica, administrativa, financiera y cambiaria.2 Luego de la citada crisis, comienza en la Argentina un proceso de recuperación, de modo que entre 2002 y 2010 el Producto Interno Bruto creció a una tasa anual del 7,6\%, el producto industrial creció a un $9,5 \%$ anual, las cuentas fiscales fueron superavitarias y se reduce el peso de la deuda pública sobre el producto (Belloni; Wainer, 2012). A su vez, tiene lugar un aumento de las exportaciones y un crecimiento del mercado interno a partir de tres medidas: tipo de cambio alto, políticas de ingreso y regulación de precios. En líneas generales, el período 2003-2008 representa una fase de recuperación en materia de exportaciones, actividad industrial, empleo, salario y consumo (Salvia, 2010).

EL contexto posterior a la crisis exhibe que el estado de la situación de los asalariados formales, en lo referente a negociaciones colectivas y monto del salario mínimo, se ha transformado, pero que en cuanto a la informalidad laboral y la inclusión de generaciones más jóvenes la problemática persiste (Halperin Weisburd, et al., 2008). De

\footnotetext{
2 En este contexto de emergencia se implementan políticas tendientes a compensar a aquellos sectores bajo condiciones de pobreza e indigencia. Dicha implementación consistió en una puesta en funcionamiento de programas de alimentación, salud, vivienda, así como del programa más significativo del momento: El Plan "Jefes y Jefas de Hogar desocupados", consistente en la transferencia de una suma de dinero (\$150) con el objetivo de mejorar las condiciones de empleabilidad de los destinatarios a través de distintos componentes que incluyen: formación profesional, terminalidad educativa y desarrollo de obras de infraestructura. El programa llega en 2003 casi a dos millones de destinatarios (Halperin Weisburd, et al., 2008).
} 
esta forma, considerando la fragmentación del mercado de trabajo como una problemática estructural -considerando que aún en 2011 el 47\% de la PEA no realizaba aportes a la seguridad social (ENAPROSS, 2014)- el Gobierno Nacional pone a circular la idea del retorno al pleno empleo como un horizonte posible, permitiendo que la población regrese al marco de las relaciones asalariadas formales, logrando así un aumento de las protecciones sociales. En este sentido, Arcidiácono (2012) desarrolla que el gobierno sostuvo discursivamente una visión "trabajocéntrica", marcando una diferencia con la visión de los 90 y que guarda cierta lógica con el carácter transitorio de los programas asistenciales. El problema reside en que, si bien los niveles de desempleo se redujeron, el empleo no registrado -como ya se dijo más arriba- no mostró un descenso tan notorio (ENAPROSS, 2014), evidenciando de esta forma la heterogeneidad estructural del mercado de trabajo en la Argentina.

Ahora bien, en este marco de heterogeneidad laboral -y subprotección social como consecuencia- en octubre de 2009, por medio del Decreto (DNU) del Poder Ejecutivo Nacional N.`1602/09 se dio origen en la Argentina a la Asignación Universal por Hijo para la Protección Social (AUH)3 cuyos destinatarios son los niños, niñas, las y los jóvenes menores de 18 años. Esta propuso la ampliación de la Ley N. 24714 que instituyó un Régimen de Asignaciones Familiares, debido a que este no incluía a los grupos familiares desocupados o que se desempeñen en la economía informal (DNU N. $\left.{ }^{\circ} 1602 / 09\right)$.

En los próximos apartados, al analizar su potencial desmercantilizador, se profundizará en las características de la AUH. A continuación, se retoman algunos puntos y perspectivas teóricas sobre el bienestar en pos de avanzar con los objetivos propuestos.

\section{Algunas líneas acerca de la provisión de "bienestar" y el "Estado de Bienestar"}

En el tránsito del siglo XIX al siglo XX, han sido centrales algunas transformaciones como los procesos de industrialización, cuya consecuencia -no única- consistió en un fuerte aumento en la población de obreros industriales instalándose en las ciudades, sedes de dicha mutación productiva. Esta concentración obrera urbana dio como resultado un incremento del pauperismo. (Comín Comín, 2007).

En el marco de esas condiciones, cabe reflexionar sobre el bienestar, lo que nos remite indefectiblemente a las formas de su provisión, pensando sobre qué orbitas recae, quién/es lo asumen, su mayor o menor mercantilización, familiarización y/o

\footnotetext{
3 La AUH Consiste en el pago de $\$ 644$ por mes, por hijo. Este monto se paga el $80 \%$ en forma directa y el $20 \%$ restante podrá ser retirado una vez al año, cuando se demuestre que el niño concurrió a la escuela y que cumplió con los controles de salud a través de la libreta nacional de seguridad social, salud y educación. En el caso de los hijos discapacitados se pagará \$2100 por mes (ANSES).

La Asignación le corresponde a todos los hijos/as de desocupados y Monotributistas sociales; y a los trabajadores no registrados y de servicio doméstico que ganen igual o menos del salario mínimo, vital y móvil. La AUH se cobra mensualmente en bancos con tarjeta magnética. (ANSES).
} 
redistribución. Podemos definir el Bienestar como ciertas capacidades para el manejo de riesgos, 4 incertidumbres, contingencias, que como ya veremos pueden organizarse analíticamente en "Regímenes". También se debe hacer alusión a la forma de concebir las necesidades. Para esto, tomamos los desarrollos de Agnes Heller (1986) sobre la teoría de la necesidad en Marx, quien describe como primer hecho histórico la creación de nuevas necesidades, no dadas en la constitución biológica, a diferencia de los animales, cuyo objeto ya está dado. Producto de determinaciones históricas para satisfacer sus necesidades humanas, el hombre lleva a cabo un proceso de creación y transformación del medio.

Ahora bien, en cuanto a la cuestión que compete a este escrito, y vinculando el bienestar con la constitución de necesidades, realizamos la pregunta acerca de cómo un grupo de necesidades se convierte en una cuestión de derechos, cómo a cierto grupo de necesidades le corresponde un grupo de satisfactores que deben ser garantizados por fuera de relaciones mercantiles. Gamallo (2012) a partir de revisar diversas perspectivas teóricas, se pregunta cómo en una sociedad donde las necesidades son infinitas, cierto grupo de ellas se constituye como dentro de una relación de derecho, constituyendo el par necesidades-satisfactores. Esto, podría decirse, se debe a que tanto las necesidades como las estructuras de riesgos no son ahistóricas ni estáticas. Martínez Franzoni (2005) lo expone claramente al elaborar las mutaciones en aquello que se concibe como bienestar.

En pos de avanzar con el desarrollo propuesto, cabe la distinción entre lo que podemos pensar y definir como "bienestar" y lo que entendemos por "Estado de Bienestar". Este último se considera en tanto intervención política del Estado para la satisfacción de necesidades (Santarsiero, 2011) que implicarían reducir esos riesgos y desprotecciones. Dentro de la formación social capitalista, el Estado de Bienestar, como desarrollo de las formas de provisión de satisfactores para la vida, tiene sus orígenes a comienzos del siglo $\mathrm{XX}$, de la mano del canciller Alemán Otto Von Bismarck. Este consistió en el otorgamiento de modo no discriminatorio del seguro social,5 rompiendo con las instituciones de beneficencia. De esta forma, el Estado comenzaba a apropiarse de diversas esferas de la producción de bienestar, transfiriendo a la esfera pública ciertas necesidades sociales a través de la conformación de los derechos sociales centrados en la figura del trabajador asalariado. Su desenvolvimiento se debe a dos factores determinantes. Por un lado, al desarrollo de la denominada "cuestión social" (Rosanvallon, 1995), en tanto serie de conflictos que se inician con la

\footnotetext{
4 Desde aquí la noción de riesgos no se considera desde el enfoque neoclásico, donde éstos recaen enteramente sobre los individuos y no son tratados como probabilidades de los colectivos (Arcidiácono, 2012).

5 El seguro social se basaba en la aplicación automática de un beneficio ante un daño como el desempleo, accidentes de trabajo, la vejez, enfermedades. La percepción del beneficio se hallaba ligada a la condición de ciudadano contribuyente y asalariado, no a la condición de pobreza.
} 
consolidación del movimiento obrero como actor social y cuyo proceso se remonta al siglo XIX. A la vez que puede ser considerado como una herramienta para la competencia política en un contexto de fuerte democratización y extensión del sufragio.

En cuanto a sus alcances y pensando en la intervención estatal para la satisfacción de las necesidades sociales:

... el Estado de Bienestar se entiende como la particular relación de pactos sociales subyacentes, arreglos de la economía capitalista y demandas de los sectores del trabajo. Estos marcos regulatorios se complementaron con medidas contracíclicas, como el gasto público deficitario para estimular la demanda solvente o medidas financieras y monetarias que se aplicaron a nivel mundial y que fueron respetadas durante toda la época de los treinta gloriosos. La economía de posguerra desarrolló una etapa expansiva y a la vez desplegó una serie de avances muy beneficiosos en términos de inclusión social y democratización del consumo (Santarsiero, 2011: 35).

Hasta aquí hemos considerado las nociones de Bienestar, necesidades y Estado de Bienestar. El paso siguiente es recuperar unas líneas sobre lo que Esping Andersen (2000) elaboró como los regímenes de bienestar. Estos son arreglos sociales que abordan de diferentes maneras las estructuras de riesgos. El autor construye una tipología de tres principales regímenes que exhiben ciertas combinaciones posibles para la provisión según recaiga este mayormente en el Estado, el mercado o la familia. El primer tipo es el liberal, el segundo el social demócrata y el tercero el conservador. El régimen liberal se remonta, filosóficamente, a la economía política británica del siglo XIX que demostraba una fe ilimitada en el funcionamiento del mercado. Este régimen intenta, desde la órbita política, minimizar el rol del Estado, individualizar los riesgos y fomentar las soluciones brindadas por el mercado, lo que resulta en un desfavorecimiento de los derechos. El régimen social demócrata remite generalmente a los países nórdicos y su consolidación se remonta a la década del 60 . El rasgo propio de este régimen, según el autor citado, es la fusión entre universalismo y un nivel de subsidios generoso, confluyendo en una socialización global de los riesgos. Los derechos se basan en la ciudadanía más que en una necesidad demostrada o en una relación laboral y el objetivo está puesto en independizar el bienestar del mercado. El régimen conservador se caracteriza fuertemente por su segmentación entre familiarismo (protección social centrada en el varón cabeza de familia y la familia como dispensadora de cuidados y responsable de sus miembros) y estatus (divisiones de estatus corporativistas que impregnan los sistemas de seguridad social) determinando diferencias en la provisión. 
La tipología anterior se recupera, ya que pensar en los regímenes de bienestar implica analizar cómo se da la combinatoria entre las distintas esferas que pueden proveer satisfactores (Estado, el mercado, las familias o la sociedad civil) y cuál de estas prima en la provisión. En este sentido, para analizar una política social, es fundamental observar qué esferas tienen mayores funciones en su aplicación y dónde recae finalmente, en mayor o menor medida, la tarea de provisión de bienestar.

Antes de abordar directamente la esfera de la desmercantilización, es de suma importancia -a los fines de este trabajo- situar el debate desde América Latina, con las particularidades que la región conlleva $y$, en segundo lugar, considerar cómo el bienestar, en tanto conjunto de satisfactores, se constituye históricamente de maneras diversas. En el continente, la idea del bienestar se asocia por lo general a su ausencia o insuficiencia, basando estos debates en las desigualdades y pobreza que padecen amplias franjas de población (Martínez Franzoni, 2005).

En este sentido, Martínez Franzoni (2005) retoma la cuestión acerca de las formas en que las definiciones sobre el bienestar se van modificando a lo largo de la historia. De esta forma, en los 50 y 60 el bienestar se vinculaba con las condiciones que conllevaban los procesos de industrialización y urbanización de las grandes ciudades. Circulaban en el continente la Teoría de la Modernización y las teorías de la marginalidad,6 otorgando diferentes explicaciones a la problemática de la pobreza y la integración social. La primera puso el énfasis en la salud y la educación como motores de integración a la pauta social urbana e industrial de la época. Esto se tradujo en la expansión de la cobertura social para la población asalariada y la expansión de la educación para la población en general.

En los 70, la discusión sobre el bienestar se centraba en torno a la satisfacción de necesidades básicas, lo que marcó el inicio de los programas dirigidos especialmente a la población pobre. En los 80 , de la mano de ajustes estructurales, se cuestionaba la deseabilidad de que la intervención estatal se dirijiese a toda la población. De esta

6 La teoría de la modernización (DESAL, CEPAL) y la teoría de la marginalidad económica (Nun, Murmis, Marín, Quijano) persiguen explicar la problemática de la marginalidad en América Latina. La Teoría de la modernización la explica en términos culturales, ya que alude a segmentos de la población en los cuales subsisten modos de vida vinculados a formas tradicionales y estructuras precapitalistas. Se describe, por parte de esta vertiente, una coexistencia entre sectores modernos de la sociedad y sectores tradicionales que aún no se encuentran adaptados/integrados a las pautas de vida y conducta de la sociedad moderna e industrial, e incluso se han descrito como una resistencia cultural para adaptarse a las nuevas pautas. Desde esta perspectiva, las causas de la marginalidad se centran en los individuos y sus propias características.

Por su parte, en la década de 60, se da un viraje en las explicaciones sobre la marginalidad. Empieza a pensarse en sus causas de manera estructural, como fenómeno que ocurre bajo las leyes de un sistema de acumulación capitalista, dentro del marco de un esquema industrializador que no alcanza a absorber la fuerza de trabajo disponible. En dicho marco, se desarrolla el concepto de "Masa Marginal", el mismo refiere a una población excedente que no es relevante para el sector hegemónico de la economía. Las relaciones de producción imperantes que se generan, son condición de posibilidad de este excedente a la vez que su existencia no es un obstáculo para su funcionamiento. 
forma, las transferencias se realizaban a la población de bajos recursos, inaugurando la estrategia de la focalización de las intervenciones. En los 90, se llevó a cabo una serie de reformas del Estado al servicio de crear las capacidades necesarias para el funcionamiento del aparato productivo. En esta línea y junto con las interpretaciones de los organismos internacionales de crédito -el Banco Mundial principalmente-, la política pública se orientó a la creación de capital humano, especialmente en educación y en salud a los sectores de más bajos recursos. La capacitación permitiría el ingreso al mercado de trabajo en pos de satisfacer las necesidades básicas (Martínez Franzoni, 2005).

Este recorrido por las formas en que el concepto va transformando su significado década tras década deja ver que no existe ningún tipo de neutralidad en su interior. Cada momento histórico define sus regímenes de bienestar a partir de las políticas públicas que pone a funcionar. En este sentido, se va a pensar la política social actual, tratando de precisar sus vinculaciones con la esfera del mercado.

\section{La desmercantilización: una esfera a considerar}

La mercantilización de crecientes aspectos de la vida es un tema central de la sociología en general y que comienza con el paso de una sociedad tradicional a una moderna. En este traspaso es que se ha creado lo que varios autores (Marx, Polanyi, Offe) dieron en llamar "mercancías ficticias", como el trabajo, la tierra y el capital (Adelantado et al., 1998). La fuerza de trabajo se ha constituido entonces, en esta mutación, en mercancía. En tanto ficticia, no es una mercancía como cualquier otra, ya que debe sobrevivir y reproducirse, por lo que no puede retirarse del mercado sin valerse de otros medios de subsistencia. Dentro de un proceso paralelo, entonces, fuerza de trabajo y necesidades humanas se constituyen como mercancías, allanando el terreno para la política social, en tanto el bienestar -como capacidades para el manejo de riesgos, incertidumbres, contingencias- se encuentra crecientemente dentro de circuitos monetarios.

De modo contrario, "La desmercantilización es, por lo tanto, un proceso con múltiples raíces; como sostuvo Polanyi, es necesaria para la supervivencia del sistema. Es también una precondición para conseguir un nivel tolerable de bienestar y de seguridad individual" (Esping Andersen, 1990: 59). En este sentido, la evolución del Estado de Bienestar exhibe las diferentes respuestas y combinatorias a las presiones de la mercancía trabajo hacia la desmercantilización. Esto no sucede en la línea de abolir la forma mercancía de la fuerza de trabajo, sino con miras a lograr que los sujetos puedan mantener una vida socialmente aceptable sin depender enteramente de la venta de su fuerza de trabajo en el mercado (Esping Andersen, 1990). Además, la desmercantilización cobra importancia como forma de atenuar el conflicto social que produce la mercantilización creciente. 
Una vez que se ha constituido la sociedad de mercado y que los satisfactores se han instalado dentro de circuitos monetarios, la desmercantilización hace alusión a las formas en que los Estados ponen a funcionar determinados andamiajes. Dichos andamiajes aseguran jurídicamente y reducen la dependencia del mercado en pos de lograr la obtención de lo necesario para la reproducción de la vida. En esta línea, "La desmercantilización se produce cuando se presta un servicio como un asunto de derecho y cuando una persona puede ganarse la vida sin depender del mercado" (Esping Andersen, 1990: 41). Esta ha sido una cuestión crucial dentro de los debates sobre la provisión de Bienestar.

Como veremos aquí, la sola presencia de esquemas de asistencia social no implica de por sí desmercantilización. En el mundo laboral, es prioritaria en tanto condición para que los trabajadores puedan asociarse y movilizarse, mientras que para los empleadores siempre es problemática ya que debilita su autoridad (Esping Andersen, 1990). Sobre la cuestión, Esping Andersen (1990) piensa en distintos países y sus regímenes para exponer casos de desmercantilización y sus distintos niveles. En esta línea, Alemania es presentado como un caso pionero en la seguridad social, aunque señala que los subsidios dependieron casi enteramente del empleo y las contribuciones. En este sentido, podemos resaltar aquí una primera y central dimensión de análisis sobre la desmercantilización, que son las reglas y precondiciones. Sostiene el autor: "... no es la mera presencia de un derecho social, sino las reglas correspondientes y las precondiciones, las que dictan hasta qué punto los programas del bienestar social ofrecen auténticas alternativas a la dependencia del mercado“" (Esping Andersen, 1990: 42). Así, reglas y precondiciones refieren a las variaciones que pueden presentar las prestaciones según la ocupación y la aportación al sistema por medio del empleo.

El modelo descripto como el más cercano a la desmercantilización es el tipo Beveridge, de subsidio a los ciudadanos, que otorga un subsidio básico e igual para todos, más allá de sus actividades, empleo u ocupaciones previas. Lo que va a decir el danés es que, si bien es un esquema más solidario, no es necesariamente desmercantilizador, ya que las personas siguen dependiendo del mercado de trabajo porque estos subsidios, por su cuantía, no constituyen una alternativa real al empleo.

A continuación, presentamos un cuadro donde se listan y describen algunas dimensiones posibles para analizar la desmercantilización de una política. Posteriormente, se discutirá si existen otras dimensiones posibles para reflexionar acerca de la esfera de la desmercantilización.

Algunas dimensiones para analizar la desmercantilización

\begin{tabular}{|l|l}
\hline Dimensiones de análisis & Características
\end{tabular}




\begin{tabular}{|c|c|}
\hline Reglas y precondiciones & $\begin{array}{l}\text { La prestación debe ser igual para todos, } \\
\text { sin depender del empleo, actividad, } \\
\text { desempeño u ocupación previa. Las } \\
\text { prestaciones no deben variar según los } \\
\text { tipos de actividad en el mercado de trabajo } \\
\text { ni los grados de aportación. }\end{array}$ \\
\hline $\begin{array}{l}\text { Normas que rigen el acceso y salida de } \\
\text { las personas a los subsidios. }\end{array}$ & $\begin{array}{l}\text { Tiene mayor potencial desmercantilizador } \\
\text { si es de fácil acceso y no se tiene en cuenta } \\
\text { el desempeño previo en el trabajo u otras } \\
\text { actividades y no requiere comprobación de } \\
\text { necesidades. A la par, el potencial } \\
\text { desmercantilizador aumenta si los } \\
\text { programas no poseen una duración } \\
\text { limitada, marcando un período para la } \\
\text { "salida" o "graduación". }\end{array}$ \\
\hline $\begin{array}{l}\text { Cuantía de la prestación/Reposición de } \\
\text { ingresos. }\end{array}$ & $\begin{array}{l}\text { Para que sea realmente } \\
\text { desmercantilizadora, el monto de la } \\
\text { prestación debe representar una verdadera } \\
\text { alternativa al empleo. Además, el monto de } \\
\text { los subsidios debe mantenerse al nivel del } \\
\text { costo de vida. }\end{array}$ \\
\hline Garantía Jurídica & $\begin{array}{l}\text { Que la prestación se realice como un } \\
\text { asunto de derecho garantizando } \\
\text { determinados satisfactores que han sido } \\
\text { aceptados como necesidades para una vida } \\
\text { digna. }\end{array}$ \\
\hline
\end{tabular}

Fuente: Elaboración propia en base al texto de Esping Andersen: "Los tres mundos del Estado de bienestar" (1990)

\section{Otros aspectos para pensar la relación entre las políticas sociales y la desmercantilización: el caso de la AUH}

Retomando su descripción, la AUH cubre a los niños, niñas y adolescentes de hasta 18 años y sin límite de edad cuando se trate de personas con discapacidad. La prestación establece un ingreso común por hijo (hasta un total de cinco hijos) a familias que se encuentren trabajando en el sector informal de la economía o cuyo ingreso no supere el salario mínimo vital y móvil. En cuanto a la nacionalidad, se exige que los niños y adultos sean argentinos, naturalizados o residentes legales por un período no inferior a tres años (ANSES, 2013), quedando exceptuados los migrantes que no puedan demostrar la permanencia requerida en el país. Además, permanecen por fuera quienes 
se encuentren dentro del régimen de monotributo (con excepción del monotributo social), quienes se encuentren dentro del régimen de autónomos, quienes no cuenten con Documento Nacional de Identidad, quienes cuenten con un salario superior al mínimo vital y móvil, quienes perciban otros planes o programas (con excepción del Programa de Ingreso Social con Trabajo, conocido como Argentina Trabaja, Jóvenes con Más y Mejor trabajo, PROGRESAR) o prestaciones de la seguridad social, independientemente del tipo de riesgo que busque cubrir. Finalmente, se encuentran excluidos los privados de libertad (como también aquellas personas internadas en instituciones de salud mental y con discapacidad psicosocial) (Arcidiácono, Gamallo, Straschnoy, 2013).

El cobro de la asignación queda sujeto al cumplimiento de ciertas condicionalidades, en materia de salud y educación. Estas se entienden como determinadas exigencias a las que se ven sometidas las familias para percibir la transferencia. De esta forma, requiere de la acreditación anual de escolarización y controles de salud en el caso de los niños (ANSES, 2013). Mensualmente, se abona el $80 \%$ de la asignación, mientras que el $20 \%$ restante es retenido por ANSES y abonado una vez que se demuestre el cumplimiento de las condicionalidades.

Ahora bien, en el presente apartado se va a discutir la posible desmercantilización de la $\mathrm{AUH}$ en las prácticas de sus destinatarios, teniendo en cuenta lo recuperado sobre la provisión de bienestar y lo atinente al potencial7 desmercantilizador de las políticas sociales. Para esto, se retoma el cuadro presentado anteriormente, a los fines de abordar ahora un caso específico.

La AUH y la esfera de la desmercantilización

\begin{tabular}{|l|l|}
\hline Dimensiones de Análisis & Características de la AUH \\
\hline Reglas y precondiciones & La política en cuestión presenta reglas y \\
precondiciones para el acceso, \\
determinando que la prestación no sea \\
para todos ni en cualquier condición. Las \\
personas con menores a cargo deben \\
estar desempleadas o trabajando de \\
manera informal, no debe superarse, en \\
el caso de estar empleados formalmente, \\
el salario mínimo vital y móvil, entre \\
otras, de forma que esta dimensión de la
\end{tabular}

7 Sobre el impacto de la $\mathrm{AUH}$, generalmente se considera la reducción de la pobreza e indigencia, así como las consecuencias a largo plazo, producto de la implementación de condicionalidades. Desde aquí se intenta pensar efectos "potenciales" de las políticas sociales actuales. En una línea similar -en términos de considerar efectos potenciales- Garganta y Gasparini (2012) realizan un estudio para considerar los potenciales efectos de la $\mathrm{AUH}$ en la informalidad laboral. 


\begin{tabular}{|c|c|}
\hline & $\begin{array}{llll}\text { política no contribuye a la } \\
\text { desmercantilización. }\end{array}$ \\
\hline $\begin{array}{l}\text { Normas que rigen el acceso y salida de } \\
\text { las personas a los subsidios }\end{array}$ & $\begin{array}{l}\text { En este punto la AUH tampoco cumple } \\
\text { un rol desmercantilizador, ya que } \\
\text { requiere de comprobación de } \\
\text { necesidades, así como contiene normas } \\
\text { para la permanencia, que son las } \\
\text { denominadas "condicionalidades". A su } \\
\text { vez, no presenta una duración limitada, } \\
\text { siendo un punto a favor de la política. La } \\
\text { "graduación" o salida del programa tiene } \\
\text { lugar si el núcleo familiar accede al } \\
\text { mercado de trabajo formal obteniendo } \\
\text { un ingreso superior al salario mínimo. }\end{array}$ \\
\hline $\begin{array}{l}\text { Cuantía de la prestación/Reposición de } \\
\text { ingresos. }\end{array}$ & $\begin{array}{l}\text { La AUH no constituye una alternativa al } \\
\text { empleo, como el mismo decreto que le } \\
\text { dio origen afirma, constituye "un } \\
\text { paliativo para alivianar las condiciones de } \\
\text { vida de los sectores más postergados". } \\
\text { Sin embargo, la prestación se actualizará } \\
\text { semestralmente, cumpliendo con el } \\
\text { requisito de reposición de ingresos. }\end{array}$ \\
\hline Garantía jurídica & $\begin{array}{l}\text { La AUH es un decreto y no una ley. A } \\
\text { diferencia de una ley, el decreto es } \\
\text { elaborado y emitido por el Poder } \\
\text { Ejecutivo, tiene menor rango que una ley } \\
\text { y, generalmente, se realiza en situaciones } \\
\text { de urgencia. Para Esping Andersen este } \\
\text { último punto -la garantía jurídica- se } \\
\text { vincula con que la prestación se ejerza } \\
\text { como un derecho, lo que entra en } \\
\text { contradicción con la aplicación de ciertas } \\
\text { condicionalidades para su cobro, ya que } \\
\text { un derecho, por definición, no debería } \\
\text { estar condicionado. }\end{array}$ \\
\hline
\end{tabular}

Fuente: elaboración propia.

En líneas generales, no podría afirmarse que la política en cuestión "desmercantiliza". Esta no cumple con muchas de las dimensiones que hemos listado, pero, además, 
debemos considerar que los programas y asignaciones que son una transferencia monetaria presentan determinadas "consecuencias" que deben constituirse como una nueva dimensión de análisis. Para desarrollar esta dimensión, se toman en cuenta las formas en que la bancarización que conlleva el acceso a estos programas implica consideraciones como la "inclusión financiera",8 además de, por ejemplo, discursos presidenciales de la región donde se resalta el papel de las nuevas estrategias para el tratamiento de la pobreza. La pregunta central es si estas "consecuencias" de los proyectos e implementaciones de política pública no deberían ser un aspecto ineludible para analizar su rol desmercantilizador, considerando que en muchos casos terminan constituyendo una "vuelta al mercado".

La desmercantilización como tal, implica la no dependencia del mercado de trabajo para alcanzar una vida en condiciones socialmente aceptables. En este sentido, la AUH es un complemento a los ingresos que las familias pueden obtener por su desempeño en un puesto de trabajo informal, o el único ingreso en el caso de desempleo. De todos modos, el objetivo de la política no fue desde su origen lograr la desmercantilización plena de las familias destinatarias. Lo que el mismo decreto afirma es que será "un paliativo importante" para su situación de pobreza, significará más dinero en los bolsillos de los sectores postergados, que no es el fin de la pobreza, sino una respuesta reparadora, y que la solución estructural se encuentra en el crecimiento económico y en la creación constante de puestos de trabajo (DNU N. ${ }^{\circ}$ 1602/09).

La AUH consiste, de este modo, en una política que intenta 'alivianar' la situación de pobreza, a la vez que forma parte desde su institucionalidad del Régimen de Asignaciones Familiares, siendo un aspecto novedoso, ya que las políticas de atención a la pobreza, en general, no han tenido por objetivo la desmercantilización total de un conjunto de satisfactores, sino que atendieron situaciones puntuales a partir de las definiciones de pobreza y urgencia de una determinada coyuntura.

8 La inclusión financiera ha sido trabajada en un escrito presentado en las Séptimas Jornadas de Jóvenes Investigadores del IIGG, a partir del análisis de documentos del Banco Central de la República Argentina y un documento del International Research Development Center (IRDC). En ambos se comienza a observar los alcances de los Programas de Transferencias Monetarias Condicionadas (PTMC). En varios documentos la AUH es considerada como un PTMC. Más allá de esa discusión, se pretende recuperar la mirada benevolente que hay en el ingreso de los sectores mas pobres al mercado financiero.

En medio de la informalidad en la que se desenvuelven los hogares pobres, facilitar su acceso a los mercados financieros -y con esto a otros mercados-, significa convertirlos en ciudadanos económicos. Desafortunadamente, el acceso a servicios financieros es, en el mejor de los casos, limitado y en general nulo para la población pobre" (Maldonado, Moreno, Giraldo Pérez, Orjuela, 2011:8). Estos autores nombran una serie de motivos por los cuales las estrategias de inclusión financiera pueden resultar provechosas para los hogares pobres. En primer lugar, el crédito podría convertirse en un instrumento útil para quienes cuentan con empleos, en general, informales, por lo que sus flujos de ingresos son irregulares. En segundo lugar, los productos financieros podrían colaborar en la solución de situaciones imprevistas, como desastres naturales, muerte de familiares, desempleo, etc. Por último, se supone que el acceso a mecanismos de ahorro formal, favorecerían la acumulación de activos por parte de estos sectores, permitiendo que puedan materializar inversiones productivas (Maldonado, Moreno, Giraldo Pérez, Orjuela, 2011). 
Ahora bien, para analizar la última dimensión -propuesta por este escrito-, es decir, las 'consecuencias' de esta política social, se recuperan brevemente trabajos que exhiben algunos aspectos y análisis resultantes de la aplicación de un PTCI.9 Se pretende hacer mención acerca de la forma en que el funcionamiento de estas transferencias y su masividad devienen en incentivos al consumo. De este modo, la vinculación entre políticas sociales y consumo se vuelve ineludible a la hora de analizar la posible desmercantilización de un programa.

En primer lugar, Lavinas (2014) refiriéndose a esta vinculación, teniendo en cuenta el alcance que tienen las transferencias de ingreso, no solo en América Latina, sino más allá de sus fronteras10, sostiene:

Tal y como afirmó enfáticamente un funcionario del FMI en un seminario organizado conjuntamente por la Fundación Friedrich Ebert Stiftung y la OIT, «no hay economía dinámica si no hay consumidores». En este programa, la lucha contra la pobreza y el avance del capitalismo financiero se han fusionado (Lavinas, 2014: 83).

Lo que se pretende situar es la diferencia entre las instituciones del bienestar, las formas estatales de asegurar el bienestar, cierto nivel de cobertura mínimo independiente de la venta de la fuerza de trabajo en el mercado y políticas que resultan en inyecciones de circulante en las economías. Si bien los sujetos se hallan en una sociedad de mercado y deben concurrir a él para obtener los bienes necesarios para su reproducción, se debe hacer especial hincapié en cómo estas formas de política social en Argentina denominadas de 'inclusión'-, impactan en el mercado, así como en las cualidades que asume dicho impacto.

Desde otra perspectiva, De Sena y Scribano (2014) mencionan cómo las prácticas estatales llevadas adelante en la actualidad como PTCI devienen en -tal como ellos lo

9 Si bien algunas autoras consideran que la AUH no consiste en un PTCI (Pautassi; Arcidiácono; Straschnoy, 2013), desde aquí, y de acuerdo con De Sena, es considerada un PTCI ya que: "Por un lado en cuanto al rol de la mujer, se evidencia que este tipo de política conlleva una visión maternalista que considera a las madres como corresponsables junto con el Estado en la consecución de ciertos objetivos sociales, como la superación de la pobreza. Se antepone la responsabilidad individual de demostrar la asistencia escolar y los controles de salud para conseguir los bonos a la responsabilidad del Estado de garantizar los servicios sociales que aseguren derechos humanos como son la educación y la salud de los niños, niñas y adolescentes (CEPAL, 2013). En segundo lugar, en tanto es resultado de un Decreto de Necesidad y Urgencia. Y, por último, resulta una intervención de corte focalizado y masivo, dados los criterios de selección de los beneficiarios y las beneficiarias" (De Sena, 2014: 114).

10 En primer lugar, Lavinas (2014) sostiene que, mientras en América Latina, para 1997, solo tres países aplicaban estos programas, para 2011 ya se aplicaban en 18 países. Para 2008, los tenían 30 países, entre ellos India, Turquía, Nigeria, Camboya, Filipinas y Burkina Faso. (Lavinas, 2014) Esto visibiliza que esta modalidad ya atravesó las fronteras de América Latina.

En América Latina se estima que han llegado a concentrar, para 2011, aproximadamente 129 millones de destinatarios (Stampini y Tornarolli, 2012). 
definen- consumos compensatorios. Estos autores retoman discursos de presidentes de América Latina que refieren al lugar preponderante del consumo en el desarrollo y la mirada benevolente sobre su intensificación por medio de transferencias a los quintiles más bajos de la población, aumentos en el salario mínimo, subvenciones, como formas de lograr la activación del mercado interno. Desde allí:

Estilos de consumo, aumento de las clases consumidoras, transferencias compensatorias, eliminación de tensiones, son expresiones claras de cómo las administraciones estatales depositan en la expansión del consumo el rol clave de: evitar conflictos, refuncionalizar la participación de millones de sujetos en el mercado y redefinir a los ciudadanos en tanto consumidores (De Sena y Scribano, 2014: 73).

Wilkis (2014), por su parte, desarrolla la centralidad del mercado del crédito en la economía popular a partir de una ligazón particular, consistente en la transformación del mercado del crédito en conjunto con la orientación de las políticas públicas a partir del año 2003. En primer lugar, se visualiza una considerable tendencia en alza de los créditos al consumo entre 2003 y 2012, pasando de 4503 millones en 2003 a 106313 millones de pesos en 2012, exhibiendo también una gran diversificación en su oferta, que va desde préstamos bancarios, tarjetas de crédito, hasta préstamos de agencias financieras y comercios minoristas (Wilkis, 2014). Estas transformaciones lograron convertir a las clases bajas en sujetos de crédito, ampliando dicho mercado y otorgándole nuevas características y matices, aumentando su heterogeneidad.

En segundo lugar, sobre la línea que siguen las políticas públicas del período en Argentina:

Estas buscaron mejorar los niveles de ingresos de los sectores populares por medio del aumento de la cobertura de las transferencias condicionadas de dinero, de la extensión de la cobertura previsional y la preservación de los niveles de empleo. El gobierno fue proponiendo una nueva interpretación sobre el dinero público que circulaba hacia los sectores más relegados. Intentaba pasar desde una política de contención, en la que el dinero era transferido para prevenir un deterioro de las condiciones de vida, a una política de rehabilitación en la que el dinero era enmarcado en el uso positivo que tenía no solo para sus receptores, sino para el conjunto de la economía (Wilkis, 2014: 231).

En la misma línea, a partir del informe sobre créditos al consumo elaborado por PROCELAC (2013) se puede observar como estos impactan de manera creciente en los sectores populares. Así, 
El sistema financiero penetró la economía de los sectores populares mediante los denominados préstamos en efectivo o vía emisión de tarjetas de crédito a partir de la masificación del consumo. La generalización de estos instrumentos financieros lejos de tener un carácter integrador o democratizador reprodujeron las diferencias sociales que operan en otros ámbitos de la sociedad (PROCELAC, 2013: 9).

La reproducción de estas diferencias se debe a las altas tasas de interés y desprotección que presentan estos préstamos. Al momento de 'contratar' un préstamo se le exige al deudor que firme un pagaré, que en caso de incumplimiento, se ejecuta de manera inmediata a través de un juicio ejecutivo.

El citado documento refiere una serie de datos provenientes del Banco Central de la República Argentina que exponen el crecimiento de los créditos al consumo, mostrando que para el año 2003 los préstamos personales representaban un 66,9\% del total de préstamos en pesos a personas físicas, incrementándose a un 73,2\% en 2012. Las razones se describen así:

Este crecimiento de los créditos para el consumo en cualquiera de sus variantes tiene un anclaje en la realidad económica y social que atraviesa la Argentina desde el año 2003, momento en que se concluyeron cinco años consecutivos de caída del PBI. La condición de posibilidad de este fenómeno debe rastrearse entonces en el aumento de los ingresos que hicieron que la clase media recupere su capacidad de consumo, en el incremento del empleo y en las políticas redistributivas implementadas, entre las que se encuentra la transferencia de dinero vía políticas sociales por parte del Estado nacional y las provincias hacia los sectores de menores ingresos (PROCELAC, 2013: 182).

En sumatoria, toda la evidencia recolectada y brevemente expuesta, intenta repensar la desmercantilización de la política social, pensando en las dimensiones propuestas por Esping Andersen y, a la vez, tratando de incorporar nuevos ejes para considerarla. En este sentido, las 'consecuencias', como han sido nombradas hasta el momento, es la dimensión que debería -al menos- ser tenida en cuenta. La construcción y ejecución de políticas públicas y sus 'efectos' son el sustrato para pensar y re-pensar el vínculo entre política social, consumo y estructura social.

\section{Reflexiones finales}

El recorrido realizado en este trabajo constituyó un ejercicio muy fructífero. Si bien la articulación que se pretende plantear es, al menos, compleja; se buscó recoger distintas perspectivas y aportes para pensar la problemática, considerando las dimensiones clásicas de análisis e incorporando nuevos aspectos para pensar la desmercantilización.

Como ya se sostuvo en el apartado anterior, es dificultoso analizar el posible impacto desmercantilizador de una política "paliativa contra la pobreza", tal como se sostiene 
desde el decreto que le dio origen. La dificultad aumenta porque desde su institucionalidad es incorporado al Régimen de Asignaciones Familiares. Como ya se dijo, esto implica un avance significativo, junto con la actualización periódica de su monto y la no limitación en la inscripción. En líneas generales, las precondiciones y las condicionalidades resultan ser el "punto flojo" de la política, entrando en contradicción con la lógica de un derecho social, debido a que para su goce no debería ser necesaria ninguna contraprestación. Esto puede decirse en cuanto a las dimensiones del análisis clásico que se listan en el cuadro.

Pensar sociológicamente las políticas sociales implica un trabajo de revisión acerca de las condiciones donde esas políticas se despliegan tratando de observar su afectación mutua. Partiendo de la recursividad entre estructura social y políticas sociales, es menester resaltar que, en una sociedad donde tanto disfrute como desarrollo económico se asocian al consumo, la política pública se encuentra encaminada en dirección a su fomento e incentivo.

Se ha buscado resaltar un proceso de estructuración social que, si bien no es el objeto principal de este trabajo, se vincula directamente. $Y$ es tan importante esta vinculación porque nos obliga a establecer, estudiar y releer los nexos entre consumo y política, a cuestionar las identidades que se construyen entre consumo y producción, como justificación política a un modo de vida centrado en la adquisición de bienes. Esto atraviesa a todos los sectores sociales, no solo a aquellos que son destinatarios de Programas de Transferencia de Ingresos. Lo que se intenta resaltar es el modo en que el consumo es un rector de la vida social, que atraviesa las aspiraciones, prácticas y sensaciones de los sujetos, así como también la política pública.

\section{Bibliografía}

ACUÑA, C.; KESSLER, G.; y REPETTO, F. (2002). "Evolución de la política social Argentina en la década de los noventa: cambios en su lógica, intencionalidad y en el proceso de hacer la política social". Proyecto Self Sustaining Community Development in Comparative Perspective. Disponible en:

http://lanic.utexas.edu/project/laoap/claspo/dt/0003.pdf Fecha de consulta: 25/02/2014 ADELANTADO, J., NOGUERA, J., RAMBLA, X., y SAEZ, L. (1998). Las relaciones entre estructura y políticas sociales: una propuesta teórica. En Revista Mexicana de Sociología Número 3, México: Universidad Autónoma de México.

ANSES (2013). "Asignación Universal por Hijo para Protección social en perspectiva. Decreto 1602/09" Boletín Cuatrimestral. 1.er cuatrimestre 2013. Bs. As. Observatorio de la Seguridad Social. Administración Nacional de Seguridad Social. 
http://observatorio.anses.gob.ar/archivos/publicaciones/OBS-000253\%20\%20Bolet\%C3\%ADn\%20Cuatrimestral\%20de\%20la\%20Asignaci\%C3\%B3n\%20Uni versal\%20por\%20Hijo\%20para\%20Protecci\%C3\%B3n\%20Social.\%20III\%20Cuatri mestre\%20de\%202013.pdf

ARCIDIACONO, P. (2012). La política del "mientras tanto". Programas sociales después de la crisis 2001-2002. Buenos Aires: Biblos.

ARCIDIÁCONO, P. GAMALLO, G.; STRASCHNOY, M. (2013). ¿Consolidación de un sistema de protección social no contributivo en Argentina? el Plan Nacer, la Asignación Universal por Hijo y la Asignación por Embarazo. En CLÉRICO, L., RONCONI, L. y ALDAO, M. (Coords.) Tratado de Derecho a la Salud. (ISBN: 978950-20-2526-1). Buenos Aires: Abeledo Perrot.BELLONI, P.; WAINER, A. (2012). La Argentina en la Posconvertibilidad: ¿Un nuevo modelo de desarrollo? Un análisis de los cambios y las continuidades en el intercambio comercial. Documento de trabajo $N^{\circ} 23$, Área de economía y tecnología, FLACSO. Disponible en:

http://cipibicargentina.org.ar/docs/DT23_Intercambio\%20comercial_\%20Wainer_Belloni .pdf

BORGHINI, N.; BRESSANO, C.; LOGIUDICE, A. (2012). La actualidad de la política social. ¿Retorno del estado que no fue o nueva matriz de la asistencia? La revista del CCC [PDF]. Septiembre / Diciembre, $n^{\circ} 16$. Disponible en Internet:

http://www.centrocultural.coop/revista/exportarpdf.php?id=348. ISSN 1851-3263.

COMÍN COMÍN, F. (2007). El surgimiento y desarrollo del Estado de Bienestar. En Salvador Salort i Vives y Ramiro Muñoz Haedo (Coord.), El Estado de Bienestar en la encrucijada. Editores: Universidad de Alicante. España.

CHAHBENDERIAN, F. (2014). Reflexiones en torno a los Programas de Transferencias Monetarias Condicionadas y los Créditos al consumo. En: Las políticas hechas cuerpo y lo social devenido emoción: lecturas sociológicas de las políticas sociales. Bs. As: Estudios Sociológicos Editora; Córdoba: Universitas- Editorial Científica Universitaria.

DE SENA, A. y SCRIBANO, A. (2014). Consumo Compensatorio: ¿Una nueva forma de construir sensibilidades desde el Estado? En Revista Latinoamericana de Estudios sobre Cuerpos, Emociones y Sociedad - RELACES, №15. Año 6. Agosto noviembre 2014. Córdoba. ISSN:1852.8759. pp. 65-82. Disponible en: http: //www.relaces.com.ar/index.php/relaces/article/view/335

DETTANO, A., LAVA, M. (2014). Entablando vinculaciones entre la sociedad de consumo, las sensibilidades y las políticas sociales desde el sur global. En Boletín Científico Sapiens Research Vol. 4(2)-2014 / pp: 27-32 / ISSN-e: 2215-9312. Disponible en: http://www.sapiensresearch.org/images/pdf/v4n2/V4N2_Sociologando_1.pdf

DE SENA, A. (2014). Las políticas hechas cuerpo y lo social devenido emoción: lecturas sociológicas de las políticas sociales. Bs. As: Estudios Sociológicos Editora; Córdoba: Universitas- Editorial Científica Universitaria. 
ENAPROSS (2014): Diagnóstico de la informalidad laboral a partir de los datos relevados por la Encuesta Nacional de Protección y Seguridad Social. Ministerio de Trabajo, Empleo y Seguridad Social de la Nación.

ESPING-ANDERSEN, G. (1990). The three worlds of capitalist welfare. Oxford University Press. London.

ESPING ANDERSEN, G. (2000). Fundamentos sociales de las economías postindustriales. Barcelona: Ariel.

GAMALLO, G. (2012). "Desmercantilización del bienestar. Aproximaciones críticas a los derechos sociales y a la política social. El caso de la educación." Congreso 2012 de la Asociación de Estudios Latinoamericanos (LASA), San Francisco, California, 23 al 26 de mayo.

GRASSI, E.; ALAYÓN, N. (2005). Condiciones de empleo y pobreza en la Argentina. Las consecuencias de la política neoliberal de los años 90. Rev. Fac. Cien. Ecón. Univ. Nac. Mayor de San Marcos 10(25), (p. 111-128)

HALPERIN WEISBURD, L. LABIAGUERRE, J., DE SENA, A., GONZÁLEZ, M., HOREN, B., MÜLLER, G., QUIROGA， L., VILLADEAMIGO, J., CHARVAY, C., HALPERIN, C., LABIAGUERRE, E., PUJOL BUCH, V. y CHAHBENDERIAN, F. (2008). Políticas sociales en la Argentina. Entre la ciudadanía plena y el asistencialismo focalizado en la contención del pauperismo. Cuadernos del CEPED N 10. (Centro de Estudios sobre Población, Empleo y Desarrollo). Facultad de Ciencias Económicas. UBA. Buenos Aires, Argentina.

HELLER, A. (1986). Teoría de las necesidades en Marx. Barcelona: Ediciones Península.

HINTZE, S.; DANANI, C. (2013). Seguridad social y condiciones de vida la protección social en la Argentina entre 2002 y 2012. En: Voces en el Fénix, Año 4, N.²3.

ISUANI, A. (1991). "Bismarck o Keynes: ¿Quién es el culpable? (Notas sobre la crisis de acumulación)"- en Isuani, Ernesto, Lo Vuolo, Ruben y Tenti, Emilio (Comps.) "El Estado de Bienestar: la crisis de un paradigma" CIEPP/Miño Dávila editores, Buenos Aires 1991

MALDONADO, J. H.; MORENO, S.; GIRALDO PÉREZ, I.; BARRERA ORJUELA, C. A. (2011). Programas de transferencias monetarias condicionadas e inclusión financiera: oportunidades y desafíos en América Latina. Bogotá: IDRC-CRDI.

MARTINEZ FRANZONI, J. (2005). Regímenes de Bienestar en América Latina: consideraciones generales e itinerarios regionales. Revista Centroamericana de Ciencias Sociales, FLACSO Vol. 4 N. ${ }^{\circ} 2$.

PROCELAC (2013). "Créditos para el consumo. Análisis del fenómeno socioeconómico y su impacto en los sectores populares". Procuraduría de criminalidad económica y lavado de activos. Ministerio Público Fiscal. Procuración General de la Nación.

SALVIA, A. (2010). De marginalidades sociales en transición a marginalidades económicas. En N. Cohen y C. Barba (Coord.), Los desafíos de la cohesión social en América Latina. (pp. 107-135). Buenos Aires: CLACSO. 
SANTARSIERO, L. (2011). Necesidades, bienestar e intervención social del estado: ejes de debate conceptual para el desarrollo de políticas sociales. Enfoques, v. 23, n. 1, jun. 2011 Disponible en:

http://www.scielo.org.ar/scielo.php?script=sci_arttext\&pid=S1669-

27212011000100003 Accedido en 1/11/ 2015.

SCRIBANO, A. (2013). Una aproximación conceptual a la moral del disfrute. Normalización, Consumo y espectáculo. Revista Brasileira de Sociología da emoción. Vol. 12, N.³6, pp. 738-750.

STAMPINI, M.; TORNAROLLI, L. (2012). The growth of conditional cash transfers in Latin America and the Caribbean: did they go too far? (No. 49). IZA PolicyPaper.

TITMUSS, R. (1974). What is the social policy? EN: Social Policy: An Introduction. Routledge

LAVINAS, L. (2014). La asistencia social en el siglo XXI. En New Left Review N84. (pp. 748) Disponible en: http://newleftreview.es/authors/lena-lavinas Fecha de consulta: $15 / 11 / 2014$

WILKIS, A. (2014). Sociología del crédito y economía de las clases populares. En Revista Mexicana de Sociología 76, N. 2 (abril-junio, 2014): (pp. 225-252). México, D.F. Universidad Nacional Autónoma de México-Instituto de Investigaciones Sociales. ISSN: 0188-2503/14/07602-03. 